[Agr. Biol. Chem., Vol. 32, No. 4, p. 501 506, 1968]

\title{
Studies on Flavor Components of Roasted Barley
}

\section{Part II. The Major Volatile Carbonyl Compounds}

\author{
By Pao-shui Wang, Hiromichi Kato and Masao Fujimaki \\ Department of Agricultural Chemistry, Faculty of Agriculture, The University of Tokyo \\ Received October 16, 1967
}

\begin{abstract}
From the volatiles of roasted barley or those formed during roasting of barley, furfural, 2-methylbutanal, 2-methylpropanal, 3-methylbutanal, 2,3-pentanedione, ethylglyoxal and pyruvaldehyde were isolated and identified as their mono- or bis-2,4-dinitrophenylhydrazones.

Removal of the carbonyl compounds from the volatiles resulted in a loss of the characteristic aroma of roasted barley.
\end{abstract}

Hrdlicka et al. ${ }^{11}$ reported that a number of aldehydes and ketones were present in the volatiles of toasted oats. The carbonyls certainly play an important role in the flavor of toasted or roasted foods. Roasted barley contains delicious flavor, but little has been done to identify the specific compounds associated with its typical flavor. One of the authors ${ }^{23}$ have reported that 1 ) the most favorable aroma developed when barley was roasted at about $160^{\circ} \mathrm{C}$ for twenty minutes, 2) the part of aleurone was more reactive than any other parts, and 3 ) during the roasting process the color gradually changed to brown and carbon dioxide occurred. The present paper deals with the results on the qualitative investigations of the volatile carbonyl compounds from roasted barley.

\section{MATERIALS AND METHODS}

Raw barley. General size, produced in Saitama prefecture, Japan.

Authentic 2,4-dinitrophenylhydrazone (2,4DNPH) of carbonyl compounds. Furfural, 2-methylpropanal, 3-methylbutanal, 2,3-pentanedione and pyruvaldehyde were obtained from commercial sources. Each compound was treated with 2,4-dinitrophenyl-

1) J. Hrdlǐcka and G. Janiček, Nature, 201, 1223 (1964).

2) P.S. Wang and Y. Sakurai, J. Japanese Soc. Food and Nutrition, (Abstract paper) 19, 116 (1966). hydrazine solution prepared from $2 \mathrm{~g}$ of the crystalline hydrazine and 1 liter of $2 \mathrm{~N}$ hydrochloric acid. 2,4DNPHs of furfural, 2-methylpropanal and 3-methylbutanal were recrystallized from $80 \%$ ethanol; bis2,4-DNPHs of 2,3-pentanedione and pyruvaldehyde were recrystallized from nitrobenzene. Authentic 2methylbutanal was prepared as follows: the mixture of $\mathrm{L}$-isoleucine and ninhydrin was heated and the resulting volatile was passed through the 2,4 -dinitrophenylhydrazine solution, 31 and the precipitated 2,4DNPH was recrystallized from $80 \%$ ethanol. Authentic bis-2,4-DNPH of ethylglyoxal was provided by Dr. T. Kurata.4

Trapping method of carbonyl compounds

a) Trapping of volatile carbonyls from ground roasted barley. Fig. I shows the apparatus used for trapping carbonyl compounds as their $2,4-\mathrm{DNPH}$ derivatives. Raw barley was roasted at about $160^{\circ} \mathrm{C}$ for $20 \mathrm{~min}$. After cooling to room temperature, the roasted barley was finely ground and put into the round bottom flask as shown in Fig. 1. To $1 \mathrm{~kg}$ of roasted barley powder, 2.3 liters of distilled water was added with stirring. The sample was maintained at $45^{\circ} \mathrm{C}$ throughout the experiment with the aid of a muffeheater. Nitrogen was bubbled into the sample and the inner pressure was reduced to about $20 \mathrm{~mm}$ $\mathrm{Hg}$ with an aspirater. Most of the carbonyls were trapped and precipitated in trap $\mathrm{C}$, small amounts of them in trap $D$, but trap $E$ remained unchanged

3) W. S. Fones, J. Am. Chem. Soc., 76, 1377 (1954).

4) T. Kurata and Y. Sakurai, Agr. Biol. Chem., 31, 177 (1967). 


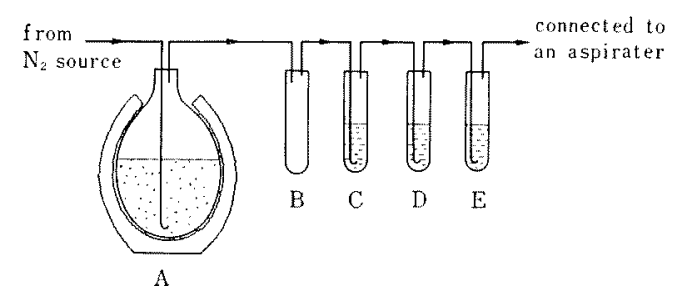

FIG. 1. Trapping Apparatus Used for Ground Roasted Barley.

A: Distillation flask with a muffe heater

B: Buffering bottle

$\mathrm{C}, \mathrm{D}$ and $\mathrm{E}$ : Trapping bottle, containing $2,4-$ dinitrophenylhydrazine solution

throughout the experiment, indicating that the carbonyls in volatiles were completely removed. 2,4DNPH derivatives thus obtained were filtered and washed with $2 \mathrm{~N}$ hydrochloric acid repeatedly until yellow color disappeared in the filtrate, washed again with water and dried over calcium chloride in a vacuum desiccator. Approximately $30 \mathrm{mg}$ of the 2,4DNPH mixture was obtained from each experiment.

b) Trapping of volatile carbonyls formed during roasting barley. In order to investigate the volatile carbonyls formed during roasting of barley, the volatiles formed were bubbled directly into the 2,4-dinitrophenylhydrazine solution as shown in Fig. 2. The electric roaster was made by coiling nichrome wire around the quartz glass tube of $54 \mathrm{~cm} \times 2.5 \mathrm{~cm}$ i.d. Raw barley was mealed to about 16 mesh just before use and the powder was put in the electric roaster. The inner temperature of roaster was maintained at
$160 \pm 5^{\circ} \mathrm{C}$ and the flow rate of air was $50 \sim 60 \mathrm{ml}$ per min. Soon after roasting commenced, precipitates began to appear in the first trap (trap $\mathbf{C}$ ), and no precipitate appeared in the last trap (trap E) throughout the experiment. After roasting for $2 \mathrm{hr}$, to the condensate in buffering bottle (trap B) was added 10 volumes of the saturated solution of 2,4dinitrophenylhydrazine in $2 \mathrm{~N}$ hydrochloric acid, and the precipitates were combined with those in trap $\mathrm{C}$ and D, and then treated similarly as (a).

Fractionation of the 2,4-DNPH derivatives by column chromatography. The mixture of $2,4-\mathrm{DNPH}$ derivatives obtained above was fractionated by column chromatography on silica-gel. About $220 \mathrm{~g}$ of silicagel of $100 \sim 120$ mesh particle range was mixed with the mixture of benzene and chloroform (3:1), and then packed into glass column of $3.5 \mathrm{~cm}$ i.d. Approximately $120 \mathrm{mg}$ of the mixture of $2,4-\mathrm{DNPH}$ derivatives was dissolved in $30 \mathrm{ml}$ of chloroform and transferred to the top of the column, then developed with the mixture of benzene and chloroform $(3: 1)$. The colored bands of the 2,4-DNPH were collected separately on elution, then evaporated to dryness.

Thin-layer chromatography of 2,4-DNPH deriva* tives. Each fraction of the 2,4-DNPH derivatives obtained from column chromatography was still so contaminated that they were isolated by preparative thin-layer chromatography until a completely single band was attained, 2 or 3 times repetition being necessary for this purpose. A glass plate coated $0.25 \mathrm{~mm}$ in thickness with Kieselgel $\mathrm{G}$ (Merck) was activated for $2 \mathrm{hr}$ at $110^{\circ} \mathrm{C}$, on which a solution of 2,4-DNPH dissolved in chloroform or ethyl acetate

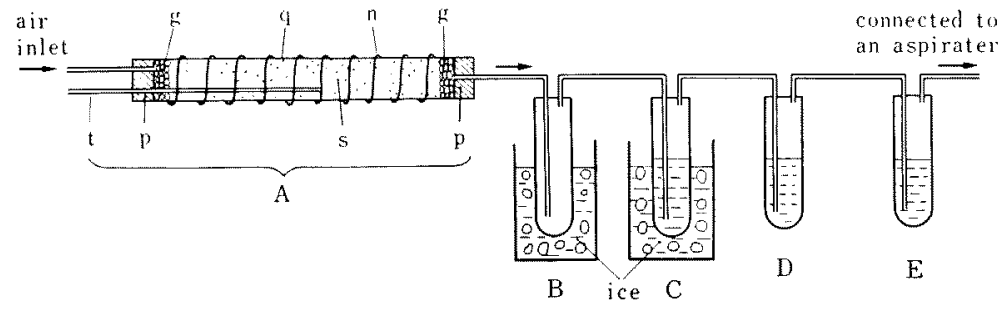

FIG. 2. Apparatus Used for Trapping Volatile Carbonyl Compounds Formed during Roasting Process.

A: An electric roaster, made by a quartz glass tube $(q)$, nichrome wire $(n)$, thermometer $(t)$, and silicone rubber plug $(p)$. The sample $(s)$ was put in the roaster with the glass wool $(\mathrm{g})$.

B: A buffering bottle

$\mathrm{C}, \mathrm{D}$ and $\mathrm{E}$ : Trapping bottle, containing 2,4-dinitrophenylhydrazine solution 
was dropped to form a thick line. Development was performed by the usual ascending method using the following solvent systems: solvent system I, benzenechloroform, $3: 1(\mathrm{v} / \mathrm{v})$; solvent system II, ethyl acetaten-hexane, I: $3(\mathrm{v} / \mathrm{v})$; solvent system III, petroleum ether $\left(\mathrm{bp} 30 \sim 70^{\circ} \mathrm{C}\right)$-diethyl ether, $10: 1(\mathrm{v} / \mathrm{v})$; solvent system IV, nitrobenzene-chloroform-benzene, $1: 2: 3$ $(\mathrm{v} / \mathrm{v})$; solvent system $\mathrm{V}$, benzene-dioxane-ethyl acetate, $20: 0.08: 0.4(v / v)$. After development, each component was extracted by chloroform and evaporated in a rotary evaporator. The residues were crystallized from $80 \%$ ethanol, chloroform or nitrobenzene.

Ultra-violet and visible spectrometry. The spectrum of each single component of 2,4-DNPH was obtained in chloroform solution with a EPS-3T Hitachi Recording Spectrophotometer.

Infrared spectrometry. Infrared spectra of these single components were obtained in nujol mull with a JASCO Model IR-S Infrared spectrophotometer.

Melting point determination. This was carried out on a heat-stage microscopical apparatus. Melting points were uncorrected.

Regeneration of carbonyls from their 2,4-DNPH derivatives. Regenerations were performed according to the method of Ralls ${ }^{5}$ with a slight modification. Two to $5 \mathrm{mg}$ of $2,4-\mathrm{DNPH}$ derivatives were triturated with 3 times their weight of $\alpha$-ketoglutaric acid and the mixtures were heated. By using a gastight syringe, the regenerated carbonyl vapor was taken from the hot tube immediately after regeneration and injected into the gas chromatograph.

Gas chromatography. Two $\mathrm{ml}$ of the regenerated carbonyl vapors were analyzed with Shimadzu Model DC-1C Gas Chromatograph equipped with a hydrogen flame detector using a 10 feet $\times 3 \mathrm{~mm}$ i.d. stainless steel column packed with $10 \%$ (w/w) tricresyl phosphate on Diasolid M, 40 60 mesh. Nitrogen was the carrier gas and flow rate was 35 to $40 \mathrm{ml}$ per minute. Column temperature was maintained at $30 \pm 2^{\circ} \mathrm{C}$.

\section{RESULTS AND DISCUSSION}

The precipitates of 2,4-DNPH derivatives obtained by the trapping procedure described above were examined preliminarily with thinlayer chromatography before their separation by column chromatography. Thin-layer chromatogram of the mixture of $2,4-\mathrm{DNPH}$ ob-

5) J. W. Ralls, Anal. Chem., 32, 332 (1960). tained from the volatiles of roasted barley with solvent system I showed seven main spots. As shown in Fig. 3, 2,4-DNPH from the volatiles formed during roasting barley also showed qualitatively the same pattern. Accordingly, the main carbonyls of roasted barley and those formed during roasting barley were considered to be qualitatively similar. Upon spraying the ethanolic solution of potassium hydroxide, spots $1 \sim 3$ gave deep brown color, indicating that they were 2,4-DNPH from monocarbonyls. Chromatography on a column of silica-gel with solvent system I yielded seven main bands which were collected separately and termed fractions $1 \sim 7$, fraction 1 being eluted first. Thin-layer chromatography of these fractions showed that each fraction contained more or less impurities and fractions 1 and 7 contained two or more components. Fraction 1 was separated into two fractions, $1 \mathrm{~A}$ and $1 \mathrm{~B}$, by preparative thinlayer chromatography with solvent system II

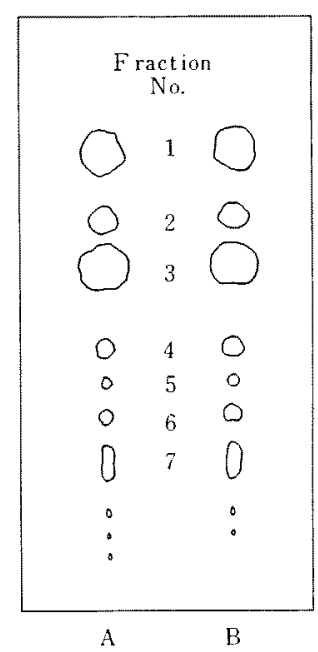

FIG. 3. The Thin-Layer Chromatogram of the Mixture of 2,4-DNPH Developed with Solvent System I.

A: $2,4-\mathrm{DNPH}$ of carbonyls from ground roasted barley

B: 2,4-DNPH of carbonyls formed during roasting barley 


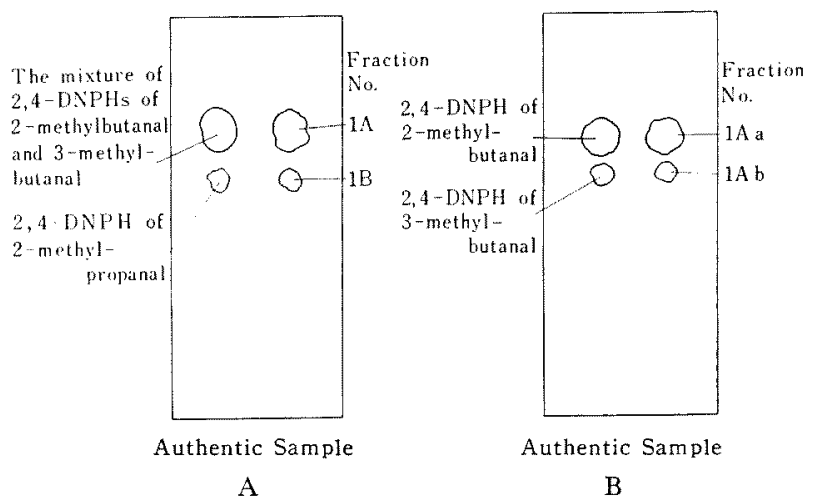

FIG. 4. The Thin-Layer Chromatograms of Fraction 1 in Comparison with Authentic 2,4-DNPHs of Carbonyl Compounds with Solvent System II (A) and Solvent System III (B).

TABle I. IDENTIfication Data on 2,4-DNPH DeRIVATIVES OF CaRbonyl Compounds Isolated FROM THE VOLATILES OF ROASTED BARLEY

\section{Compounds}

Fraction $1 \mathrm{Aa}$

2-Methylbutanal 2,4-DNPH

Fraction $1 \mathrm{Ab}$

3-Methylbutanal 2,4-DNPH

Fraction 1B

2-Methylpropanal 2,4-DNPH

Fraction 2

Furfural 2,4-DNPH (syn)

Fraction 3

Furfural 2,4-DNPH (anti)

Fraction 4

2,3-Pentanedione

bis-2,4-DNPH

Fraction 5

(Unidentified)

Fraction 6

Ethylglyoxal

bis-2,4-DNPH

Fraction $7 \mathrm{~A}$

Pyruvaldehyde bis-2,4-DNPH

Fraction $7 \mathrm{~B}$

(Unidentified) $R f^{*} \lambda_{\max }$
$(\mathrm{m} \mu)$

$1.28 \quad 358$

$1.28 \quad 358$

$1.28 \quad 358$

$1.28 \quad 358$

$1.28 \quad 357$

1.28 357

$1.13 \quad 374$

$1.13 \quad 374$

$1.00 \quad 388$

$1.00 \quad 388$

$0.78 \quad 396$

$0.78 \quad 440(s)$

$\begin{array}{ll}0.78 & 396 \\ & 440(\mathrm{~s})\end{array}$

$0.70 \quad 398$

$0.60 \quad 396$

$\begin{array}{ll} & 440(s) \\ 0.60 & 396\end{array}$ 396
$440(s)$

0.51395

0.51395 $440(s)$

$0.51 \quad 412$ $450(\mathrm{~s})$
$\mathrm{Mp}\left({ }^{\circ} \mathrm{C}\right)$ Mixed $\mathrm{mp}\left({ }^{\circ} \mathrm{C}\right)$

Elementary analyses**

* Rf: $R f$ of 2,4-DNPH of component/Rf of 2,4-DNPH of furfural (anti) with benzene-chloroform $3: 1$.

** (): Calculated value 
(Fig. 4A), Fraction 1B was a single component which yielded yellow needles on crystallization from hot $80^{\circ}$ ethanol. It was identified as 2,4-DNPH of 2-methylpropanal, by thin-layer chromatography, ultra-violet and infra-red absorption spectra (Fig. 5-I), mixture melting point, and elementary analysis (Table I). Similarly, fraction 2 was identified as the syn form of furfural 2,4-DNPH, and fraction 3 as the anti form of furfural 2,4-DNPH (Table I, Figs. 5-II and 5-III). Fraction 1A gave only one spot on thin-layer chromatogram developed with solvent system I or II. However, on development with solvent system III, fraction $1 \mathrm{~A}$ was separated into two fractions, $1 \mathrm{Aa}$ and $1 \mathrm{Ab}$ (Fig. 4-B). Both fractions $1 \mathrm{Aa}$
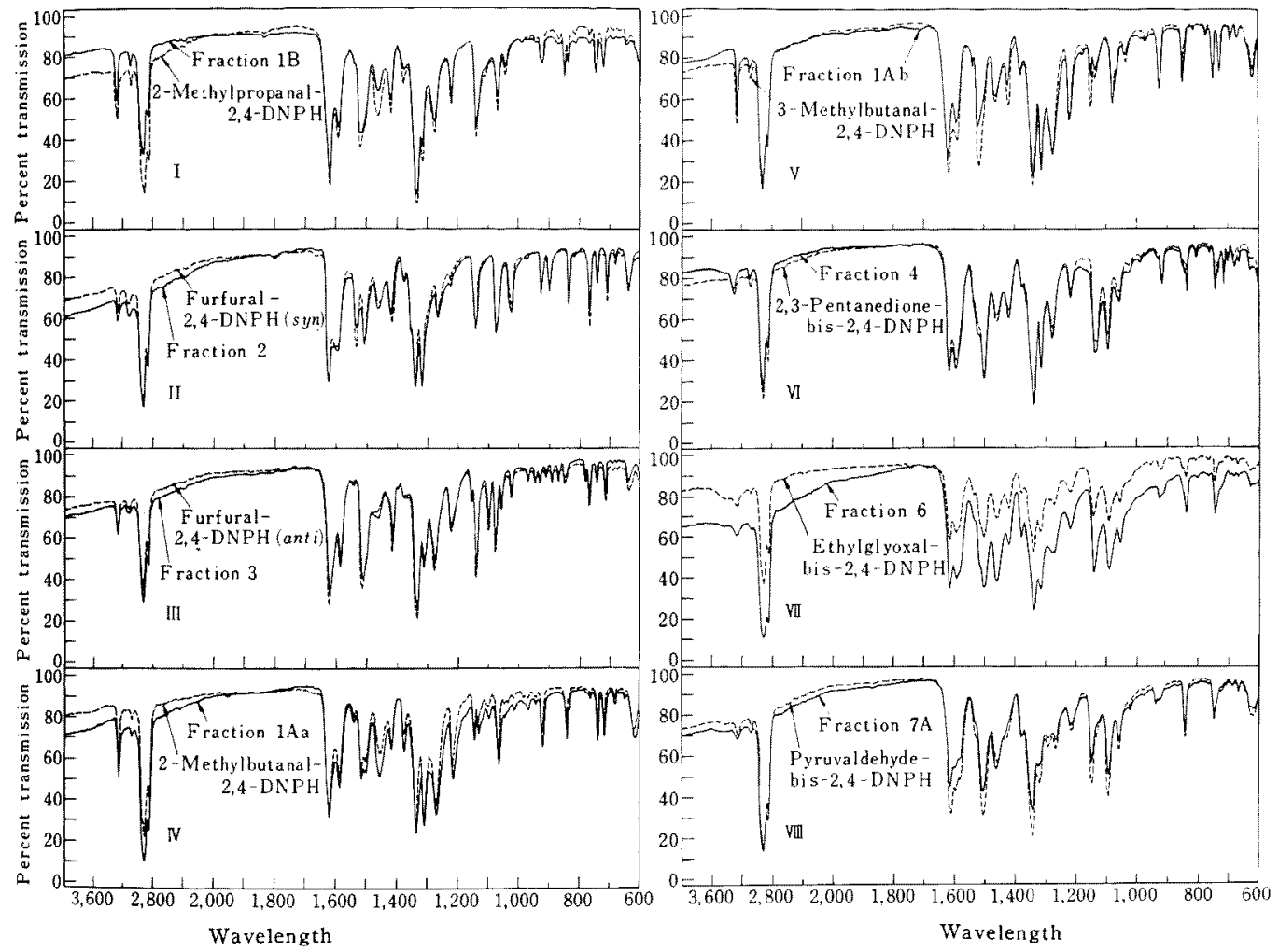

FIG. 5. Infrared Spectra of Fractions $1 \sim 7$ in Nujol Mull.

\section{- Isolated}

-.---- Authentic

I: 2-Methylpropanal 2,4-DNPH (fraction 1B)

II: Furfural 2,4-DNPH, syn form (fraction 2)

III: Furfural 2,4-DNPH, anti form (fraction 3)

IV: 2-Methylbutanal 2,4-DNPH (fraction $1 \mathrm{Aa}$ )

V: 3-Methylbutanal 2,4-DNPH (fraction $1 \mathrm{Ab}$ )

VI: 2,3-Pentanedione bis-2,4-DNPH (fraction 4)

VII: Ethylglyoxal bis-2,4-DNPH (fraction 6)

VIII: Pyruvaldehyde bis-2,4-DNPH (fraction 7A) 
and $1 \mathrm{Ab}$ yielded yellow crystals from 80,0 ethanol. The behavior on thin-layer chromatography, and ultra-violet and infrared absorption spectra of fraction 1Aa (Table I, Fig. 5IV) were identical with those of 2-methylbutanal 2,4-DNPH. Similarly, fraction $1 \mathrm{Ab}$ was identical with 3-methylbutanal 2,4-DNPH. Further evidence was given by gas chromatography. The retention times of the regenerated vapors of fractions $1 \mathrm{Aa}$ and $1 \mathrm{Ab}$ were same with those of the authentic 2methylbutanal and 3-methylbutanal, respectively.

On spraying the ethanolic potassium hydroxide, fractions $4 \sim 7$ gave violet color, being characteristic of the bis-2,4-DNPH derivatives of compounds having adjacent carbonyl groups. ${ }^{6}$ In order to remove the impurities contained in fractions $4 \sim 7$, fractions 4 and 5 were thin-layer chromatographed with solvent system IV, then crystallized from nitrobenzene, and fraction 6 with solvent system I, then crystallized from chloroform. Fraction 7 was separated into two fractions, $7 \mathrm{~A}$ and $7 \mathrm{~B}$ by thin-layer chromatography with solvent system V. Both $7 \mathrm{~A}$ and $7 \mathrm{~B}$ were crystallized from nitrobenzene. Fractions 4, 6 and $7 \mathrm{~A}$ yielded orange-colored crystals and were identified by thin-layer chromatography, ultra-violet and infrared absorption spectra, and mixture melting point, as bis-2,4-DNPHs of 2,3-pentanedione, ethylglyoxal and pyruvaldehyde, respectively. (Table I, Figs. 5-VI, 5-VII and 5-VIII). The crystals obtained from fractions 5 and $7 \mathrm{~B}$ were very small in amounts and identification could not be attained.

These components identified are the major carbonyls produced when barley are roasted under the conditions described. However, other components are also present, because the mixture of 2,4-DNPH derivatives obtained above was not completely soluble in chloroform, benzene or ethyl acetate. The com-

6) C. Neuberg and E. Strauss, Arch. Biochem., 7, $211(1945)$. ponents, which were present in much lower concentrations and ran faster than the 2,4DNPH derivatives of the C5-aldehydes with solvent system I, may be longer-chain carbonyls.

Among the carbonyls identified above, the amounts of monocarbonyls were much more than those of the dicarbonyls and furfural was most abundant.

Furfural, 2,3-pentanedione, ethylglyoxal and pyruvaldehyde are probably the degradation products of the carbohydrates of barley. 2Methylpropanal, 2-methylbutanal and 3-methylbutanal could be derived from valine, isoleucine and leucine, respectively, by Strecker degradation." Among the identified carbonyl compounds, 2,3-pentanedione and ethylglyoxal have been identified for the first time in this paper in the volatiles of cooked or roasted foods.

All the carbonyls detected in the volatile of roasted barley have pungent odors when each of them exists alone and in concentrated state. But when they are mixed and in some low concentrations, they give a somewhat favorable odor. However, on removing the carbonyl compounds by passing the volatiles from roasted barley through the 2,4-dinitrophenylhydrazine solution, the characteristic delicious aroma of roasted barley was lost and changed to somewhat unfavorable odor. This fact indicates that the carbonyl compounds play an important role in the flavor of roasted barley. After removal of carbonyl compounds from volatiles of roasted barley there remains sulfur compounds and others which may also play some roles in the flavor of roasted barley, and these will be elucidated in the following study.

Acknowledgement. The authors are grateful to Professor Yosito Sakurai, Japan Women's University, for his valuable suggestions.

7) A. Schonberg and R. Moubacher, Chem. Revs., 50, $267(1952)$. 